andere Menschen $\mathrm{zu}$ belästigen, dürfte sich daher erhöhen.

Auch im Bereich des Polizei- und Ordnungsrechts wurden durch die Klarstellung letzte Unstimmigkeiten, wie auf Stalker zu reagieren sei, beseitigt.

Sönke Gerhold ist Diplom-Jurist (Universität Kiel).

\section{Fußnoten}

1) Vgl. BR-Drs. 551/04

2) Vgl. Gesetzentwurf des Bundesrates vom 18.03.2005 (BR-Drs. 551/04) und den Gesetzesentwurf des BMJ vom 10.08.2005 (BT-Drs. 15/5410).
3) Im BRatE wurde auf eine Handlung abgestellt, die abstrakt "geeignet ist, einen Menschen" und nicht konkret das Opfer zu beeinträchtigen. Vgl. zur Kritik an den ersten Entwürfen auch: Stefan Caspari, http://www. drb.de/?http://www.drb.de/pages/html/stellung/st-stalking.html, Stand 20.02.2007.

4) Vgl. auch Dagmar Freudenberg, Vorschläge des Deutschen Juristinnenbundes für ein sinnvolles Vorgehen gegen Stalking, NK 3/05, S. $84 \mathrm{ff}$

5) Vgl. Begründung zum Gesetzentwurf der Bundesregierung; http://dip.bundestag.de/btd/16/ 005/1600575.pdf.
6) Vgl. Tröndle/ Fischer, § 203, Rn. 27 m.w.N.

7) Vgl. Tröndle/ Fischer, § 292, Rn. 11 ff.; Schönke/ Schröder Eser/ Heine, § 292, Rn. 5.

8) BT-Drs. 16/575; http://dip. bundestag.de/btd/16/005/1600 575.pdf.

9) BT-Drs. 16/575; http://dip. bundestag.de/btd/16/005/1600 575.pdf.

10) Vgl. zu diesem Absatz: BT-Drs. 16/575; http://dip.bundestag. de/btd/16/005/1600575.pdf.

11) Vgl. auch die Beispielsfälle zu eingestellten Verfahren bei Volkmar von Pechstaedt, in: Stalking, Möglichkeiten und Grenzen der Intervention;
Hrsg. Julia Bettermann; Seite 151 bis 156 .

12) Urteil des LG Kiel vom 03. 03. 2006, AZ.: V Ns 18/06; Beschluss des Schleswig-Holsteinischen OLG vom 28.06.2006, AZ 2 Ss 70/06.

13) Urteil des LG Kiel vom 03. 03. 2006, AZ.: V Ns 18/06; Beschluss des Schleswig-Holsteinischen OLG vom 28.06.2006, AZ 2 Ss 70/06.

14) Beschluss des BVerfG vom 27.09.2006, AZ 2 BvR $1603 /$ 06.

15) Vgl. http://www.drb.de/?http: //www.drb.de/pages/html/stellung/st-stalking.html.

\title{
Mindeststandards für
} den Jugendstrafvollzug

C as BVerfG hat dem Gesetzgeber mit seinem Urteil vom 31. Mai 2006 aufgegeben, bis Ende 2007 den Vollzug der Jugendstrafe auf eine gesetzliche Grundlage $\mathrm{zu}$ stellen. Durch die Föderalismusreform sind nun die Länder für die Regelung des Strafvollzugs zuständig. Diese Ausgangssituation macht die Formulierung einheitlicher Mindeststandards für den Jugendstrafvollzug um so notwendiger. Die Häftlingsmorde von Siegburg und - drei Jahre vorher - Ichterhausen machen eindringlich die Wichtigkeit humaner Strafvollzugstandards deutlich.

Guter (Jugend-)Strafvollzug ist immer auch eine Ressourcenfrage. Wir sind der Überzeugung, dass der allgemeinen Sicherheit am besten gedient ist, wenn die begrenzten Ressourcen des (Jugend-)Strafvollzugs auf die wirklich schwerwiegenden Fälle konzentriert werden. Neben der Jugendstrafe steht der Jugendstrafjustiz ein breites Spektrum an Reaktionsmöglichkeiten zur Verfügung. Diese müssen genutzt werden. Rückfallkriminalität würde so besser vermieden und die Integration der Betroffenen besser gefördert.

\section{Eigenständige Jugendstraf- vollzugsgesetze}

Der Jugendstrafvollzug muss in einem eigenen, vollständigen Gesetz geregelt werden. Das Bundesverfassungsgericht hat ausführlich die Besonderheit des Jugendstrafvollzugs begründet und daraus gefolgert, dass das Strafvollzugsgesetz nicht analog auf den Vollzug der Jugendstrafe angewendet werden kann. Die geforderte Eigenständigkeit geht verloren, wenn die unterschiedlichen Vollzugsarten in einem Gesetz geregelt werden. Durch gesetzesinterne Querverweise verlieren die Ge- setze zudem erheblich an Verständlichkeit.

\section{Vollzugsziel ist die Resozia- lisierung (Wiedereingliede- rung, Integration)}

Der Jugendstrafvollzug ist an dem Ziel auszurichten, den Gefangenen $z u$ befähigen, ein Leben in Freiheit ohne erneute Straffälligkeit zu führen. Dadurch dient der Vollzug zugleich der Sicherheit der Allgemeinheit (so hat es auch ausdrücklich das Bundesverfassungsgericht festgehalten). Sicherheit wird letztlich durch Rückfallverhinderung erreicht, die Rückfallverhinderung aber wird nicht dadurch optimiert, dass im Zweifel der Geschlossenheit und Restriktion Vorrang eingeräumt wird. Deshalb ist in der Gesetzesfassung darauf zu achten, dass das Resozialisierungsziel nicht durch andere Vollzugsziele oder -aufträge konterkariert wird, die den Anschein von Gleichrangigkeit erwecken.

\section{Umfassende Beteiligung der Gefangenen}

Die Gefangenen haben das Recht, an allen sie betreffenden Angelegenheiten beteiligt $\mathrm{zu}$ werden (Art.12 VN-Kinderrechtskonvention). Die Förder\& Erziehungsplanung ist daher unter aktiver Beteiligung des Gefangenen zu erarbeiten. Die Gefangenen sollen in die Lage versetzt werden, aktiv an der Gestaltung ihres Vollzugsalltags mitzuwirken.

\section{Elternrechte wahren}

Die verfassungsrechtlichen Elternrechte (Art.6 GG) werden durch den Vollzug der Jugendstrafe zwar eingeschränkt, aber nicht suspendiert (vgl. BVerfGE 107, 104, 119 = DVJJ-J 2003, 68, 71). Gerade in erzieherischen Belangen wie bspw. der Aufstellung der Förder- und Erziehungspläne ist den Eltern daher eine Mitsprachemöglichkeit einzuräumen. Sie sind nicht 
nur von wichtigen Vollzugsentscheidungen (insbesondere der Aufstellung, Änderung und Fortschreibung der Förder- und Erziehungspläne, Verlegungen usw.) zu informieren, sondern wo möglich bereits an den Entscheidungsprozessen zu beteiligen. Dies gilt auch für die Entlassungsvorbereitung.

\section{Keine unbestimmte Pflicht} zur Selbst-Resozialisierung

Der Gefangene unterliegt den im Gesetz genannten konkretisierten Einzelpflichten und ist in deren Rahmen auch zur aktiven Mitwirkung angehalten. Eine allgemeine Pflicht des Gefangenen, „an der Erreichung des Vollzugszieles" (seiner Resozialisierung) mitzuwirken, ist inhaltlich zu unbestimmt, praktisch nicht handhabbar, nicht willkürfest (weil Pflichtverletzungen Disziplinarmaßnahmen oder den Ausschluss von Vergünstigungen zur Folge haben) und daher verfassungswidrig.

\section{Verbindliche Mitwirkung der Jugendhilfe}

Die Zuständigkeit der Jugendhilfe ist während des Jugendstrafvollzugs nicht aufgehoben, Ansprüche aus dem SGB VIII werden durch diesen nicht ausgeschlossen. Das Jugendamt spielt daher von Beginn bis Ende des Vollzugs eine wichtige Rolle. Es muss verbindlich bei der Vollzugs- und Entlassungsplanung mitwirken und bereitet so rechtzeitig die Wiedereingliederung vor. Diese aktive Beteiligung des Jugendamtes muss verbindlich in den Jugendstrafvollzugsgesetzen sowie in den Ausführungsgesetzen zum SGB VIII verankert werden.

\section{Umfassende Vernetzung des Vollzuges}

Die sozialen Dienste der Justiz, Bewährungshilfe und Jugendhilfe müssen gemeinsam an kontinuierlichen, über den Vollzug hinausgehenden Betreuungsbeziehungen zum Gefangenen arbeiten. Relevante Erkenntnisse anderer Institutionen (bspw. Psychiatrie, Gericht, Poli- zei, Jugendhilfe) sind bei der Eingangsdiagnostik und der Erstellung der Förder- und Erziehungspläne heranzuziehen, damit nicht jedes Mal von vorne begonnen werden muss. Insbesondere im Rahmen der Entlassungsvorbereitung ist vernetztes Handeln und Planen wichtig.

\section{Chancen für alle Gefangenen}

Motivationsarbeit ist selbstverständlicher Teil Sozialer Arbeit, auch im Vollzug. Wer nur mit den schon Kooperationsbereiten und -fähigen arbeiten will, verschenkt fruchtbare Einflusschancen und gibt die anderen auf. Nach dem Konzept des sog. Chancenvollzuges sollen Behandlungsmaßnahmen und Vollzugslockerungen nur den Gefangenen gewährt werden, die von sich aus kooperationsbereit sind. Er vernachlässigt dadurch seinen erzieherischen Auftrag. Ein solcher Chancenvollzug bietet keine Chancen, sondern ist Ausgrenzungs- und Sparvollzug

\section{Offenen Vollzug nutzen und ausbauen}

Für die Resozialisierung und Wiedereingliederung ist es von entscheidender Bedeutung, dass der Gefangene einen Bezug zur Außenwelt behält bzw. aufbauen kann, bevor er entlassen wird. Die Möglichkeiten des offenen Vollzuges (der in den letzten Jahren in der Praxis immer weiter reduziert wurde) und von Vollzugslockerungen, sind daher vermehrt zu nutzen. Der offene Vollzug ist daher als Regelvollzug vorzusehen. Zumindest muss dies bei kürzeren Jugendstrafen (bis 3 Jahren) und bei Selbststellern geschehen. Grundsätzlich ist er nur auszuschließen, wenn auf Grund von Tatsachen die begründete Befürchtung des Missbrauchs durch Flucht oder die Begehung von Straftaten besteht.

\section{Vollzugslockerungen \& Vollzug in freien Formen}

Vollzugslockerungen (insb. Urlaub, Freigang und Ausgang) sind für die Erreichung des Voll- zugsziels besonders wichtig. Sie sind zu gewähren, wenn verantwortet werden kann zu erproben, dass der Gefangene die Vollzugslockerungen nicht zur Flucht oder Begehung von Straftaten missbrauchen wird. Vor allem bei der Entlassungsvorbereitung ist eine zunehmende Orientierung nach „draußen“ unumgänglich.

Jugendstrafrecht und Jugendstrafvollzug sind immer wieder Vorreiter gewesen in der Erprobung neuer Methoden und Formen. Die Landes-Jugendstrafvollzugsgesetze sollten daher eine Regelung enthalten, die modellhafte Projekte des Vollzuges in freien Formen ( $\$ 91$ Abs.3 JGG) ermöglicht.

\section{Rechtzeitig mit der Entlas- sungsvorbereitung begin- nen}

Der Vollzug ist von Beginn an auf die Entlassung und Wiedereingliederung auszurichten. Spätestens sechs Monate vor der geplanten Entlassung (bei kurzen Jugendstrafen vier Monate) bereiten die Jugendstrafanstalten zusammen mit anderen Behörden und Diensten (vgl. Nrn. 6 \& 7) die Entlassung vor. Der Vollzug wird gelockert (vgl. Nr.10).

\section{Unterbringung in Einzelhaft- räumen; Wohngruppen- vollzug}

Die Häftlingsmorde von Siegburg und Ichtershausen zeigen, wie wichtig Einzelunterbringung und ausreichende Betreuung der Gefangenen sind. Jeder Gefangene hat das Recht auf Unterbringung in einem Einzelhaftraum.

Überschaubare Wohngruppen sind für soziales Lernen, die Konstituierung funktionierender Gruppen und die Vermeidung von Subkultur unverzichtbar. Den Wohngruppen ist dauerhaft festes Personal zuzuweisen; sie sollten nicht mehr als 12 Mitglieder haben.

Die Jugendstrafvollzugsgesetze sollen hierzu klare und verbindliche Aussagen machen. Dies fordert auch das Bundesverfassungsgericht in seinem Urteil vom 31.05.2006 (ZJJ 2006, S.196f).

\section{Eigenständige Jugendstraf- anstalten}

Der Vollzug der Jugendstrafe erfolgt in eigenständigen Jugendstrafanstalten. Die Angliederung an Anstalten des ErwachsenenVollzugs und der Vollzug von Jugend- und Freiheitsstrafe in einer Anstalt sind auszuschließen. Die Anstalten sollen sich zunächst (bei Übergangsfristen von maximal 10 Jahren) an einer Größe von höchstens 240 Gefangenen orientieren. Langfristig sollten kleinere dezentrale Einrichtungen angestrebt werden.

\section{Besondere Situation von jungen Frauen und Mädchen}

Mädchen und junge Frauen sind eine kleine Minderheit im (Jugend-)Strafvollzug, der oft nicht ausreichend Rechnung getragen wird. Sie sind in eigenen Anstalten oder Häusern unterzubringen.

\section{Außenkontakte fördern}

Der Austausch mit der Außenwelt durch Briefe und Telefon ist zu fördern und umfassend zu ermöglichen. Die Mindestdauer für Besuche im Strafvollzug beträgt 4 Stunden monatlich. Familiäre Kontakte sind darüber hinaus besonders $\mathrm{zu}$ fördern und dürfen nicht aus disziplinarischen Gründen eingeschränkt werden. Der Empfang von Paketen, auch mit Lebensmitteln, ist zu gewährleisten.

\section{Recht auf Bildung}

Schulangebote sind für alle Gefangenen, die keinen ausreichenden Schulabschluss haben, zu gewährleisten. Für die berufliche Bildung sind zukunftsweisende, zeitgemäße Angebote zu schaffen, die den Gefangenen reale Beschäftigungschancen vermitteln. Im Vollzug begonnene Ausbildungen können auch nach der Entlassung in der Anstalt fortgesetzt werden. 
Schule und Berufsbildung haben Vorrang vor Arbeit; dies muss sich auch in der Bezahlung ausdrücken.

\section{Sozialversicherungen}

Die Einbindung der Gefangenen in das System der Sozialversicherungen muss gewährleistet sein.

\section{Konfliktregelung vor Diszi- plinierung}

Konflikte unter Gefangenen und mit den Mitarbeitern sind im Vollzug alltäglich. Ein auf Förderung und Erziehung ausgerichteter Jugendstrafvollzug muss primär auf Konfliktregelung anstelle von Disziplinierung setzen. Die Jugendstrafvollzugsgesetze haben daher Instrumente der Konfliktregelung vorzusehen und mit Vorrang gegenüber Disziplinarmaßnahmen auszustatten.

Konflikte können auch in pädagogischen Gesprächen aufgearbeitet werden. Für darüber hinausgehende, sanktionierende Erziehungsmaßnahmen ist hingegen kein Platz, da so das reglementierte Disziplinarwesen unterlaufen würde. Das Disziplinarwesen ist umfassend $\mathrm{zu}$ regeln. Die Normierung muss die zu ahndenden Tatbestände und die zulässigen Sanktionsmaßnahmen hinreichend bestimmt regeln. Disziplinarmaßnahmen werden verhängt, wenn eine Konfliktregelung mit den Beteiligten gescheitert oder diese oder ein Erziehungsgespräch unangemessen wären was $\mathrm{zu}$ begründen ist. Eine isolierte Unterbringung (Arrest) darf allenfalls als ultima ratio vorgesehen werden; Nr. 67 der VN-Regeln zum Schutze von Jugendlichen unter Freiheitsentzug verbietet die isolierende Einzelhaft als unmenschliche und entwürdigende Behandlung. Die Praxis zeigt, dass der Jugendstrafvollzug auch ohne dieses Disziplinierungsmittel auskommen kann.
19 Keine Schusswaffen im Jugendstrafvollzug

In Jugendstrafanstalten sind das Tragen und der Gebrauch von Schusswaffen nicht zuzulassen (Nr. 65 der VN-Regeln zum Schutze von Jugendlichen unter Freiheitsentzug). Für im Bedarfsfall hingezogene externe Polizeikräfte ist der Schusswaffengebrauch in der für diese geltenden Rechtsgrundlage (Polizeigesetz) geregelt.

\section{Effektiven Rechtsschutz schaffen}

Das Rechtsschutzsystem ist effektiv im Sinne der Zielgruppe und für diese verständlich auszugestalten. Das für Rechtsmittel gegen Vollzugsmaßnahmen zuständige Gerichts muss ortsnah sein, ein rein schriftliches Verfahren (wie in $\S 109 \mathrm{ff}$ StVollzG) ist unzureichend. Der Bundesgesetzgeber (der für den gerichtlichen Rechtsschutz zuständig ist), hat daher unverzüglich ein den Vorgaben des Bundesverfassungsgericht entsprechendes Rechtsschutzsystem zu schaffen. Die Landesgesetzgeber müssen in ihren Jugendstrafvollzugsgesetzen ein effektives Beschwerde- und Widerspruchsverfahren vorsehen.

\section{Strafvollzugsbeauftragte}

Um die Beachtung und Einhaltung internationaler Standards und völkerrechtlicher Vorgaben sicherzustellen, wird in jedem Bundesland ein/e unabhängige/r Beauftragte/r für den Strafvollzug geschaffen, der/die Zugang zu allen Strafvollzugsanstalten hat und an den/die sich die Gefangenen jederzeit mit Beschwerden wenden können. Diese erfüllen die Aufgabe der nationalen Stellen zur Prävention von Folter und anderer unmenschlicher Behandlungen nach Art. 3 des Zusatzprotokolls zur Anti-Folter-Konvention.

Die Strafvollzugsbeauftragten sind mit einem ausreichenden verwaltungsmäßigen Unterbau zu versehen. Sie berichten jährlich über die Menschenrechtssi- tuation in den Anstalten. Im Rahmen der Genehmigung von Anstaltsordnungen hat er ein Anhörungs- und Rügerecht. Er wirkt bei Auswahl der Experten des Periodischen Strafvollzugsberichts mit (s. Nr. 22).

\section{Wirksame Vollzugsgestal- tung, Evaluation}

Nach dem Urteil des Bundesverfassungsgerichts haben die Länder die am Resozialisierungsgebot orientierte Wirksamkeit ihres Vollzugskonzeptes laufend $\mathrm{zu}$ überprüfen. Sie sind zur Beobachtung und Nachbesserung verpflichtet. Dazu ist es erforderlich, die notwendigen Daten zu erheben und Statistiken zu führen. Die kriminologischen und sonst geeigneten Forschungseinrichtungen sind an der Wirkungsforschung und der Vollzugsevaluation $\mathrm{zu}$ beteiligen. In regelmäßigen Abständen sind wissenschaftliche periodische Berichte über die Entwicklung des (Jugend-)Strafvollzugs vorzulegen, die konkrete Empfehlungen für die Verbesserung der Vollzugsgestaltung enthalten.

\section{Qualifiziertes und ausrei- chendes Personal}

Die Gesetze müssen eine ausreichende Personalausstattung sicherstellen. Insbesondere muss der Personalschlüssel gewährleisten, dass die Gefangenen auch am Wochenende und an Feiertagen betreut werden und ein ausreichendes Freizeitangebot vorgehalten wird. Das Personal - gleich ob Fachdienste oder AVD - ist regelmäßig fortzubilden. Supervision muss gewährleistet sein.

Schlussbemerkung: Die Etablierung der hier benannten Mindeststandards im Jugendstrafvollzug mag mit Mehrkosten gegenüber dem Status Quo des Jugendstrafvollzugs verbunden sein. Abgesehen davon, dass die Einhaltung dieser Mindeststandards durch die Verbesserung von Wiedereingliederung und Rückfallverhinderung an anderer Stelle menschliche und finanzielle Kosten spart, sind die- se Mindeststandards aus rechtsund sozialstaatlichen Gründen geboten. Wer in einem an Menschenrechten und rationaler Intervention orientierten Europa des 21. Jahrhunderts den Jugendstrafvollzug betreiben will, kommt an ihnen nicht vorbei.

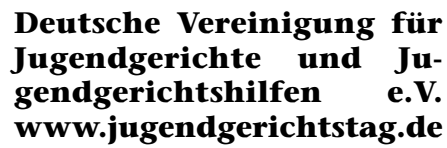

Wir rufen alle auf, diese Mindeststandards zu unterstützen. Dr. Bernd Asbrock, Bremen; Prof. Dr. Heinz Cornel, Berlin; Kristiane von den Driesch, Attendorn; Jochen Goerdeler, Hannover; Dr. Theresia Höynck, Hannover; Prof. Dr. Hans-Jürgen Kerner, Tübingen; Prof. Dr. Bernd Maelicke, Lüneburg; Prof. Dr. Heribert Ostendorf, Kiel; Prof. Dr. Christian Pfeiffer, Hannover; Prof. Dr. Hans-Joachim Plewig, Lüneburg; Dr. Helmut Pollähne, Bremen; Peter Reckling, Köln; Prof. Dr. Bernd-Rüdeger Sonnen, Hamburg; Jürgen Taege, Gelsenkirchen; Prof. Dr. Michael Walter, Köln; usw...

Verbände: DVJJ Deutsche Vereinigung für Jugendgerichte und Jugendgerichtshilfen | DBH Fachverband für Soziale Arbeit, Strafrecht und Kriminalpolitik | BAG Soziale Arbeit im Justizvollzug | ADB Arbeitsgemeinschaft Deutscher Bewährungshelfer/innen | NRV Neue Richtervereinigung usw... 